Pacific

Journal of

Mathematics

UNIFORM HYPERBOLICITY OF THE CURVE GRAPHS

BRIAN H. BOWDITCH

Volume 269 No. 2

June 2014 


\title{
UNIFORM HYPERBOLICITY OF THE CURVE GRAPHS
}

\author{
BRIAN H. BOWDITCH
}

\begin{abstract}
We show that there is a universal constant, $k$, such that the curve graph associated to any compact orientable surface is $k$-hyperbolic. Independent proofs of this have been given by Aougab, by Hensel, Przytycki and Webb, and by Clay, Rafi and Schleimer.
\end{abstract}

\section{Introduction}

Let $\Sigma$ be a closed orientable surface of genus $g$, together with a (possibly empty) finite set $\Pi \subseteq \Sigma$. Set $p=|\Pi|$. We assume that $3 g+p \geq 5$. Let $\mathscr{G}=\mathscr{G}(g, p)$ be the curve graph associated to $(\Sigma, \Pi)$; that is, the 1-skeleton of the curve complex as originally defined in [Harvey 1981]. Its vertex set, $V(\mathscr{G})$, is the set of free homotopy classes of nontrivial nonperipheral closed curves in $\Sigma \backslash \Pi$; and two such curves are deemed to be adjacent in $\mathscr{G}$ if they can be realised disjointly in $\Sigma \backslash \Pi$. These, and related, complexes are now central tools in geometric group theory and hyperbolic geometry.

In [Masur and Minsky 1999], it was shown that, for all $g, p, \mathscr{G}(g, p)$ is hyperbolic in the sense of [Gromov 1987]. In [Bowditch 2006], henceforth abbreviated [B], it was shown that the hyperbolicity constant, $k$, is bounded above by a function that is logarithmic in $g+p$. In fact, we show here that $k$ can be chosen independently of $g$ and $p$ :

Theorem 1.1. There is a universal constant, $k \in \mathbb{N}$, such that $\mathscr{G}(g, p)$ is $k$-hyperbolic for all $g, p$ with $3 g+p \geq 5$.

We will give some estimates for $k$ (though certainly not optimal) in Section 4.

Independent proofs of this result have been found by Aougab [2013], by Hensel, Przytycki and Webb [Hensel, Przytycki and Webb 2013], and by Clay, Rafi and Schleimer [Clay, Rafi and Schleimer 2013]. The proofs in these last two papers are combinatorial in nature, while Aougab's proof is based on broadly similar principles to those described here, though the specifics are different. Both this paper and [Aougab 2013] make use of riemannian geometry. The argument of [Hensel, Przytycki and Webb 2013] seems to give the best constants.

MSC2010: 20F65.

Keywords: uniform hyperbolicity, curve graph, surface. 
Given Theorem 1.1, one can also obtain uniform bounds for the bounded geodesic image theorem of [Masur and Minsky 2000]. For this, one can combine the description of quasigeodesic lines in [B] with an unpublished argument of Leininger. In fact, a more direct approach, just using hyperbolicity, has recently been found by Webb [2013].

We remark that Theorem 1.1 does not imply uniform hyperbolicity of the curve complexes (with simplices realised as regular euclidean simplices) since their 1-skeleta are not uniformly quasi-isometrically embedded - there is an arbitrarily large contraction of distances as the complexity increases.

The proof of Theorem 1.1 consists primarily of going through the arguments of [B] with more careful bookkeeping of constants. This is accomplished in Section 2 here. In Sections 3 and 4 here, we show that much of this can be bypassed. In fact, we only really need a few results from [B], notably Lemmas 1.3, 4.4 and 4.5, together with the construction of singular euclidean structures described in Section 5 thereof.

We were motivated to look again at that paper after reading some estimates in [Tang 2013] which relate distances to intersection number.

\section{Proofs}

In this section, we will prove Proposition 2.6, which, together with Proposition 3.1 of [B], implies Theorem 1.1.

We will use the following different measures of the "complexity" of $\Sigma, \Pi$, tailored to different parts of the argument: $\xi_{0}=2 g+p-4, \xi_{1}=2 g+p-1$, $\xi_{2}=2 g+p+6$. For $\alpha, \beta \in V(\varphi)$, we write $\iota(\alpha, \beta)$ for the intersection number, and $d(\alpha, \beta)$ for the combinatorial distance in the curve graph.

Lemma 2.1. If $\gamma, \delta \in V(\mathscr{G})$, with $\iota(\gamma, \delta) \leq \xi_{0}+1$, then $d(\gamma, \delta) \leq 2$.

Proof. We realise $\gamma, \delta$ in $\Sigma \backslash \Pi$ so that $|\gamma \cap \delta|=\iota(\gamma, \delta)=n$, say. Now, $\gamma \cup \delta$ is a graph with $n$ vertices and $2 n$ edges, and hence Euler characteristic $-n$. If $d(\gamma, \delta)>2$, then $\gamma \cup \delta$ fills $\Sigma \backslash \Pi$ and so this Euler characteristic must be at most that of $\Sigma \backslash \Pi$, namely, $2-2 g-p$. Thus $n \geq 2 g+p-2$. Taking the contrapositive, if $n \leq \xi_{0}+1=2 g+p-3$, then $d(\gamma, \delta) \leq 2$.

Now, Lemma 1.3 of [B] shows that if $\alpha, \beta \in V(\varphi)$ with $2 \iota(\alpha, \beta) \leq a b$ for $a, b \in \mathbb{N}$, then there is some $\gamma \in V(\mathscr{G})$ with $\iota(\alpha, \gamma) \leq a$ and $\iota(\beta, \gamma) \leq b$. Applying this $q$ times, together with Lemma 2.1, we get:

Corollary 2.2. If $q \in \mathbb{N}$ and $\alpha, \beta \in V(\varphi)$ with $2^{q} \iota(\alpha, \beta) \leq \xi_{0}^{q+1}$, then $d(\alpha, \beta) \leq$ $2(q+1)$.

Definition. By a region in $\Sigma$, we mean a subsurface, $H \subseteq \Sigma$, with $\partial H \cap \Pi=\varnothing$. A region is trivial if it is a topological disc containing at most one point of $\Pi$. An 
annulus in $\Sigma$ is a region $A \subseteq \Sigma \backslash \Pi$ homeomorphic to $S^{1} \times[0,1]$ such that no component of $\Sigma \backslash A$ is trivial.

The core curve of an annulus therefore determines an element of $V(\mathscr{G})$.

Suppose that $\rho$ is a riemannian metric on $\Sigma$. We allow for a finite number of cone singularities (which need bear no relation to $\Pi$ ). We define the width of an annulus $A \subseteq \Sigma$ to be the length of a shortest path in $A$ connecting its two boundary components.

The following lemma is a slight variation of Lemma 5.1 of [B]. We follow a similar argument, but taking more care with constants.

The proof will make use of the following notion. Let $\alpha$ be an essential nonperipheral closed curve in $\Sigma \backslash \Pi$.

Definition. A bridge (across $\alpha$ ) is an arc, $\delta \subseteq \Sigma \backslash \Pi$, with $\partial \delta=\delta \cap \alpha$ such that no component of $\Sigma \backslash(\alpha \cup \delta)$ is a disc not meeting $\Pi$.

In other words, $\alpha \cup \delta$ is an embedded $\pi_{1}$-injective theta-curve in $\Sigma \backslash \Pi$, i.e., it is the union of three arcs which meet precisely in their endpoints and are pairwise nonhomotopic relative to their endpoints.

Lemma 2.3. Let $\rho$ be a (singular) riemannian metric on $\Sigma$ with $\operatorname{area}(\Sigma)=1$. Suppose that $3 g+p \geq 5$. Suppose that there is a constant $h>0$ such that for any trivial region $\Delta \subseteq \Sigma$ we have area $(\Delta) \leq h(\text { length }(\partial \Delta))^{2}$. Then $\Sigma$ contains an annulus of width at least $\eta=\frac{1}{4} \xi_{1} \xi_{2} \sqrt{h}$.

Proof. To avoid technical details obscuring the exposition, we will relax inequalities so that they are assumed to hold up to an arbitrarily small additive constant $\epsilon>0$. Thus, for example, a "shortest" curve will be assumed to be shortest to within $\epsilon$. This will allow us, for instance, to adjust paths so that they can be assumed to avoid $\Pi$. Finally, we can allow $\epsilon \rightarrow 0$. In what follows any "curve" in $\Sigma \backslash \Pi$ will be assumed to be essential and nonperipheral, i.e., it does not bound a trivial region in $\Sigma$.

Let $\eta_{0}=1 / 4 \xi_{2} \sqrt{h}$. We claim that there are curves, $\alpha, \beta \subseteq \Sigma \backslash \Pi$ with $\rho(\alpha, \beta) \geq \eta_{0}$. Given this, we let $\phi: \Sigma \rightarrow\left[0, \eta_{0}\right]=\left[0, \xi_{1} \eta\right]$ be a 1-lipschitz map with $\alpha \subseteq \phi^{-1}(0)$ and $\beta \subseteq \phi^{-1}\left(\xi_{1} \eta\right)$. Given any $i \in\left\{1, \ldots, \xi_{1}-1\right\}$, we can find a multicurve, $\gamma_{i} \subseteq \phi^{-1}(i \eta)$, which separates $\Sigma$ into exactly two components, $S_{i}^{\alpha}, S_{i}^{\beta}$, containing $\alpha$ and $\beta$ respectively. We can assume $\gamma_{i} \cap \Pi=\varnothing$, and that $S_{i}^{\alpha} \subseteq S_{i+1}^{\alpha}$ for all $i$. These multicurves cut $\Sigma$ into $\xi_{1}$ regions $M_{i}=S_{i}^{\alpha} \cap S_{i-1}^{\beta}$ (where $M_{0}=S_{1}^{\alpha}$ and $M_{\xi_{1}}=S_{\xi_{1}-1}^{\beta}$ ). At least one of these must have a component which is an annulus (otherwise each $M_{i} \backslash \Pi$ would have negative Euler characteristic, giving the contradiction that the Euler characteristic of $\Sigma \backslash \Pi$ is at most $\left.-\xi_{1}<2-2 g-p\right)$. This annulus must have width at least $\eta$ as required.

To find $\alpha$ and $\beta$, we take $\alpha$ to be a shortest curve in $\Sigma \backslash \Pi$. We suppose, for contradiction, that if $\beta \subseteq \Sigma \backslash \Pi$ is any curve, then $\rho(\alpha, \beta)<\eta_{0}$. Let $\lambda=2 \eta_{0}$. 


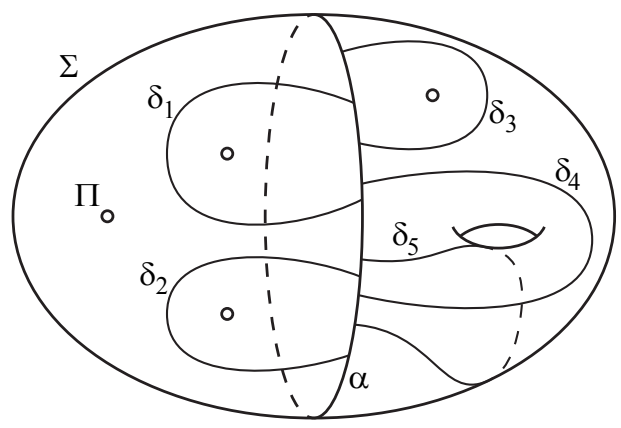

Figure 1. Example of a curve with bridges, $(g, p)=(1,4)$.

We first claim that there is a collection of disjoint bridges, $\delta_{1}, \ldots, \delta_{n}$, across $\alpha$ with length $\left(\delta_{i}\right)<\lambda$ for all $i$ and with each component of $\Sigma \backslash\left(\alpha \cup \delta_{1} \cup \cdots \cup \delta_{n}\right)$ trivial. (An example is shown in Figure 1.)

To prove this claim, let $N(\alpha, t)$ be the metric $t$-neighbourhood of $\alpha$ in $\Sigma$. Let $G(t)$ be the image of $\pi_{1}(N(\alpha, t) \backslash \Pi)$ in $\pi_{1}(\Sigma \backslash \Pi)$. Note that $G(0)$ is infinite cyclic, and $G\left(\eta_{0}\right)=\pi_{1}(\Sigma \backslash \Pi)$. As $t$ increases from 0 to $\eta_{0}, G(t)$ gets bigger at certain critical times, $t_{1}, \ldots, t_{n}$. At these times, we can suppose we have added another generator, which we can represent as a bridge, $\delta_{i}$, of length at most $2 t_{i}<2 \eta_{0}=\lambda$. Thus, inductively, $G\left(t_{i}\right)$ is supported on $\alpha \cup \delta_{1} \cup \cdots \cup \delta_{i}$. It follows that $\alpha \cup \delta_{1} \cup \cdots \cup \delta_{n}$ must fill $\Sigma \backslash \Pi$ (that is, carries all of $\pi_{1}(\Sigma \backslash \Pi)$ ), otherwise we could find a curve, $\beta$, with $\rho(\alpha, \beta) \geq \eta_{0}$. This gives us our collection of bridges as claimed.

Let $l=$ length $(\alpha)$. We now claim that $l \leq 6 \lambda$. So, suppose, to the contrary, that $l>6 \lambda$.

Given any $i$, write $\alpha=\alpha_{i} \cup \alpha_{i}^{\prime}$, where $\alpha_{i}$ and $\alpha_{i}^{\prime}$ are respectively the shorter and longer arcs with endpoints at $\partial \delta_{i}$. Thus

$$
\text { length }\left(\alpha_{i}\right) \leq l / 2 \text { and length }\left(\alpha_{i} \cup \delta_{i}\right) \leq l / 2+\lambda<l .
$$

By minimality of $\alpha, \alpha_{i} \cup \delta_{i}$ must be trivial or peripheral, i.e., it bounds a trivial region in $\Sigma$. This region must be a disc containing exactly one point of $\Pi$. Since this is true of all bridges $\delta_{i}$, we already get a contradiction if $g>0$ (and we can deduce that $l \leq 3 \lambda$ in this case). So we can assume that $g=0$, and so $\alpha$ cuts $\Sigma$ into two discs, $H_{0}$ and $H_{1}$. We have $\left|\Pi \cap H_{i}\right| \geq 2$, and we can assume that $\left|\Pi \cap H_{0}\right| \geq 3$.

Note also that, if $\alpha_{i}^{\prime} \cup \delta_{i}$ is nontrivial, then length $\left(\alpha_{i}^{\prime} \cup \delta_{i}\right) \geq$ length $(\alpha)$ and so length $\left(\alpha_{i}\right) \leq$ length $\left(\delta_{i}\right)<\lambda$.

Now $H_{0}$ must contain at least two bridges from our collection. We can assume these are $\delta_{1}$ and $\delta_{2}$. Recall that $\delta_{1} \cap \delta_{2}=\varnothing$. From the above, it follows that length $\left(\alpha_{1}\right)<\lambda$ and length $\left(\alpha_{2}\right)<\lambda$. Since $\delta_{1}$ and $\delta_{2}$ cannot cross, we must have $\alpha_{1} \cap \alpha_{2}=\varnothing$. 


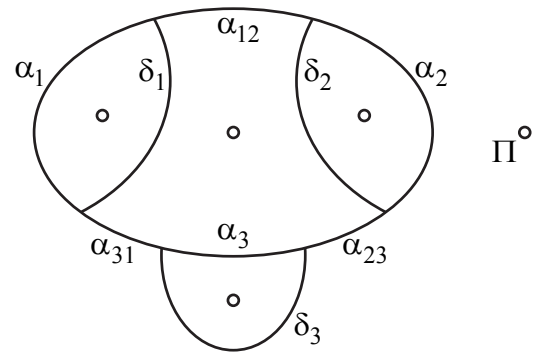

Figure 2. Picture of three bridges, $(g, p)=(0,5)$.

Now let $\delta_{3}$ be a bridge in $H_{1}$. As before, length $\left(\alpha_{3}\right) \leq l / 2$, and so for $i=1,2$, length $\left(\alpha_{i} \cup \alpha_{3} \cup \delta_{i} \cup \delta_{3}\right) \leq 3 \lambda+l / 2$. Now $\alpha_{1} \cap \alpha_{3}=\varnothing$ (otherwise $\alpha_{1} \cup \alpha_{3} \cup \delta_{1} \cup \delta_{3}$ would contain a curve of length at most $3 \lambda+l / 2<l$ ). Similarly, $\alpha_{2} \cap \alpha_{3}=\varnothing$. Now, given $i, j \in\{1,2,3\}$, let $\alpha_{i j}$ be the component of $\alpha \backslash\left(\alpha_{1} \cup \alpha_{2} \cup \alpha_{3}\right)$ between $\alpha_{i}$ and $\alpha_{j}$ (see Figure 2). Let $\theta_{i j}$ be the curve in $\Sigma$ with image $\alpha_{i j} \cup \alpha_{i} \cup \alpha_{j} \cup \delta_{i} \cup \delta_{j}$, which passes through $\alpha_{i j}$ exactly twice. Together, the curves $\theta_{12}, \theta_{23}$ and $\theta_{31}$ pass twice through each edge of $\alpha \cup \delta_{1} \cup \delta_{2} \cup \delta_{3}$, and so their lengths sum to at most $2 l+6 \lambda$. We arrive at the contradiction that the length of at least one of the $\theta_{i j}$ is at most $\frac{1}{3}(2 l+6 \lambda)<l$.

This shows that $l \leq 6 \lambda$ as claimed.

After removing some of the bridges if necessary, we can assume that at most two of the complementary components are discs not meeting $\Pi$, and so $n \leq 2 g+p$. Let $\sigma=\alpha \cup \delta_{1} \cup \cdots \cup \delta_{n}$. Thus length $(\sigma)<6 \lambda+n \lambda=(n+6) \lambda \leq(2 g+p+6) \lambda=\xi_{2} \lambda$.

Since each component of $\Sigma \backslash \sigma$ is trivial, we must have area $(\Sigma) \leq h(2 \text { length } \sigma)^{2}$ (the worst case being when $\Sigma \backslash \sigma$ is connected). But we have assumed that area $(\Sigma)=1$ and so $1<h\left(2 \xi_{2} \lambda\right)^{2}$. Now, $\lambda=2 \eta_{0}=2\left(1 / 4 \xi_{2} \sqrt{h}\right)=1 / 2 \xi_{2} \sqrt{h}$, so we arrive at the contradiction that $1<1$.

This shows that there must be a curve, $\beta$, in $\Sigma \backslash \Pi$ with $\rho(\alpha, \beta) \geq \eta_{0}$ as claimed.

In fact, the argument also applies if $(g, p)=(1,1)$. If $(g, p)=(0,4)$, we will only need to consider a special case, namely, the quotient of a euclidean torus by an involution with four fixed points. In that case, we can set $\eta=1 / 2$.

We will now set $h=1 / 2 \pi$. This gives $\eta=1 / 4 \xi_{1} \xi_{2} \sqrt{1 / 2 \pi}=\sqrt{2 \pi} / 4 \xi_{1} \xi_{2}$. As in Section 5 of [B], we define $R=\sqrt{2} / \eta$. In this case therefore, $R=(4 / \sqrt{\pi}) \xi_{1} \xi_{2}$.

Now suppose that $\alpha, \beta$ are weighted multicurves in the sense defined in [B]. (In other words, each is a measured lamination whose support is a disjoint union of curves.)

Definition. The weighted intersection number, $\iota(\alpha, \beta)$, of $\alpha$ and $\beta$ is the sum $\sum_{i, j} \lambda_{i} \lambda_{j} l\left(\alpha_{i}, \beta_{j}\right)$, where $\alpha_{i}$ and $\beta_{j}$ vary over the components of the support of $\alpha$ and $\beta$, where $\lambda_{i}$ and $\lambda_{j}$ are the respective weighting on them, and where $\iota\left(\alpha_{i}, \beta_{j}\right) \in \mathbb{N}$ is the usual geometric intersection number. 
We write $d(\alpha, \beta)=\min _{i, j}\left\{d\left(\alpha_{i}, \beta_{j}\right)\right\}$, again where $\alpha_{i}$ and $\beta_{j}$ vary over the components of $\alpha, \beta$.

Given $\gamma \in V(\varphi)$ we set $l(\gamma)=l_{\alpha \beta}(\gamma)=\max \{\iota(\alpha, \gamma), \iota(\beta, \gamma)\}$ (interpreting $\gamma$ as a one-component multicurve of unit weight). One can think of $l(\gamma)$ as describing a "length" in a singular euclidean structure arising from $\alpha$ and $\beta$ (see Section 5 of $[\mathrm{B}])$.

Lemma 2.4. Suppose that $\alpha, \beta$ are weighted multicurves with $\iota(\alpha, \beta)=1$ and $d(\alpha, \beta) \geq 2$. Then there is some $\delta \in V(\mathscr{G})$ with $l(\delta) \leq R$ such that $\iota(\gamma, \delta) \leq R l(\gamma)$ for all $\gamma \in V(\mathscr{G})$ (where $R$ is defined as above).

Proof. This is just a restating of Lemma 4.1 of [B] for this particular definition of $R$. The proof is the same. Suppose first that $\alpha \cup \beta$ fills $\Sigma \backslash \Pi$. As in Section 5 of that paper, we construct a singular euclidean surface, tiled by rectangles, dual to $\alpha \cup \beta$. The cone angles are all multiples of $\pi$, and all cone singularities of angle $\pi$ lie in $\Pi$. Thus, any trivial region, $\Delta \subseteq \Pi$, contains at most one cone point of angle less than $2 \pi$. Passing to a branched double cover over this cone point (if it exists) we are reduced to considering the case where all cone angles are at least $2 \pi$. But then the worst case is a round circle in the euclidean plane [Weil 1926] which would give area $(\Delta)=$ length $(\partial \Delta)^{2} / 4 \pi$. We can therefore set $h=2(1 / 4 \pi)=1 / 2 \pi$. Now apply Lemma 2.3, and set $\delta$ to be a core curve of that annulus. The statement then follows exactly as in [B] (at the end of Section 5 thereof). (In [B], $h$ was given inaccurately as $\pi / 2$.)

If $\alpha \cup \beta$ does not fill $\Sigma \backslash \Pi$, we get instead a singular euclidean structure on a "smaller" surface, namely a region of $\Sigma$ with each boundary component collapsed to a point. However, this process can only decrease $\xi_{1}$ and $\xi_{2}$, so we again get an annulus of width at least $\eta$. (This case is the reason we needed a version of Lemma 2.3 when $3 g+p=4$. In the case where $(g, p)=(0,4)$, note that $1 / 2$ is certainly greater than the required $\sqrt{2 \pi} / 120$.)

Given $r \geq 0$, set $L(\alpha, \beta, r)=\{\gamma \in V(\mathscr{G}) \mid l(\gamma) \leq r\}$. Note that the curve $\delta$ given by Lemma 2.4 lies in $L(\alpha, \beta, R)$.

Lemma 2.5. Suppose that $2 g+p \geq 195$. Suppose that $\alpha, \beta$ are weighted multicurves with $\iota(\alpha, \beta)=1$ and $d(\alpha, \beta) \geq 2$. Then, the diameter of $L(\alpha, \beta, 2 R)$ in $\varphi$ is at most 20.

Proof. Let $\delta$ be as given by Lemma 2.4. If $\gamma \in L(\alpha, \beta, 2 R)$, then $l(\gamma) \leq 2 R$, so $\iota(\gamma, \delta) \leq 2 R^{2}$. If we knew that $16 \iota(\gamma, \delta) \leq \xi_{0}^{5}$, then Corollary 2.2 with $q=4$ would give $d(\gamma, \delta) \leq 10$ and the result would follow.

It is therefore sufficient that $16\left(2 R^{2}\right) \leq \xi_{0}^{5}$. Recall that $R=(4 / \sqrt{\pi}) \xi_{1} \xi_{2}$, so this reduces to $32(4 / \sqrt{\pi})^{2} \xi_{1}^{2} \xi_{2}^{2} \leq \xi_{0}^{5}$, that is, $512 \xi_{1}^{2} \xi_{2}^{2} \leq \pi \xi_{0}^{5}$. In other words, we want

$$
512(2 g+p-1)^{2}(2 g+p+6)^{2} \leq \pi(2 g+p-4)^{5},
$$


which holds whenever $2 g+p \geq 195$.

We now assume that $2 g+p \geq 195$.

Recall that Lemma 4.3 of [B] states that $L(\alpha, \beta, R)$ has diameter bounded by some constant $D$ (which there, depended on $R$ ). Since $L(\alpha, \beta, R) \subseteq L(\alpha, \beta, 2 R$ ), we have now verified Lemma 4.3 of [B] with $D=20$. Recall that Lemma 4.2 of [B], more generally, placed a bound on the diameter of $L(\alpha, \beta, r)$ depending on $r$ and $R$ (specifically, diam $L(\alpha, \beta, r) \leq 2 R r+2$ ). This was used in the proof of Lemma 4.12 [B]. We can now use Lemma 2.5 above, in place of Lemma 4.2 of [B], to give a proof of Lemma 4.12 of [B] with the constant $4 D$ now replaced by 40 . We can now proceed as in [B] to prove Lemma 4.13 and Proposition 4.11 of that paper. In fact, the improvement on Lemma 4.12 allows us, respectively, to replace the constants $14 D$ by $10 D$ and $18 D$ by $14 D$, where $D=20$. Thus, the original diameter bound of $18 D$ of Proposition 4.11 of [B] now becomes 280.

Recall that Proposition 3.1 of [B] gives a criterion for hyperbolicity depending on a constant, $K$, in the hypotheses. The three clauses (1), (2), and (3) of those hypotheses were verified respectively by Lemma 4.10, Proposition 4.11 and Lemma 4.9. These respectively gave $K$ bounded by $4 D, 18 D$, and $2 D$, which we can now replace by 80,280 and 40 . In particular, we have shown:

Proposition 2.6. If $2 g+p \geq 195$, then the curve graph $G(g, p)$ satisfies the hypotheses of Proposition 3.1 of [B] with $K=280$.

For $2 g+p \geq 195$, one can now explicitly estimate $k$ from the proof of Proposition 3.1 of [B]. In fact, one can do better.

\section{A criterion for hyperbolicity}

We give a self-contained account of a criterion for hyperbolicity which is related to, but simpler than, that used in [B]. In particular, it does not require the condition on moving centres (clause (2) of Proposition 3.1 of [B]) which complicated the argument there. Essentially the same statement can be found in Section 3.13 of [Masur and Schleimer 2013], though without a specific estimate for the hyperbolicity constant arising (or the final clause about Hausdorff distance). Our proof uses an idea to be found in [Gilman 2002], but bypasses use of the isoperimetric inequality. Since this criterion has many applications, this may be of some independent interest. For definiteness, we say that a space is $k$-hyperbolic if, in every geodesic triangle, each side lies in a $k$-neighbourhood of the union of the other two.

Proposition 3.1. Given $h \geq 0$, there is some $k \geq 0$ with the following property. Suppose that $G$ is a connected graph, and that for each $x, y \in V(G)$, we have associated a connected subgraph, $\mathscr{L}(x, y) \subseteq G$, with $x, y \in \mathscr{L}(x, y)$. Suppose that: 
(1) For all $x, y, z \in V(G)$,

$$
\mathscr{L}(x, y) \subseteq N(\mathscr{L}(x, z) \cup \mathscr{L}(z, y), h) .
$$

(2) For any $x, y \in V(G)$ with $d(x, y) \leq 1$, the diameter of $\mathscr{L}(x, y)$ in $G$ is at most $h$. Then $G$ is $k$-hyperbolic. In fact, we can take any $k \geq(3 m-10 h) / 2$, where $m$ is any positive real number satisfying $2 h\left(6+\log _{2}(m+2)\right) \leq m$. Moreover, for all $x, y \in V(G)$, the Hausdorff distance between $\mathscr{L}(x, y)$ and any geodesic from $x$ to $y$ is bounded above by $m-4 h$.

Here, $d$ is the combinatorial metric on $G$, and $N(\cdot, h)$ denotes $h$-neighbourhood. Note that we can assume that $\mathscr{L}(x, y)=\mathscr{L}(y, x)$ (on replacing $\mathscr{L}(x, y)$ with $\mathscr{L}(x, y) \cup \mathscr{L}(y, x))$. Note that the condition on $m$ is monotonic: if it holds for $m$, it holds strictly for any $m^{\prime}>m$.

Proof. Given any $x, y \in V(G)$, let $\Phi(x, y)$ be the set of all geodesics from $x$ to $y$. Given any $n \in \mathbb{N}$, write

$$
f(n)=\max \{d(w, \alpha) \mid(\exists x, y \in V(G)) d(x, y) \leq n, \alpha \in \mathscr{I}(x, y), w \in \mathscr{L}(x, y)\} .
$$

In other words, $f(n)$ is the minimal $f \geq 0$ such that $\mathscr{L}(x, y) \subseteq N(\alpha, f)$ for any geodesic, $\alpha$, connecting any two vertices $x, y$ at most $n$ apart.

We first claim that $f(n) \leq\left(2+\left[\log _{2} n\right]\right) h$ (compare [Gilman 2002]). To see this, write $l=d(x, y) \leq n$ and $p=\left[\log _{2} l\right]+2$. Let $z \in V(G)$ be a "near midpoint" of $\alpha$; that is, it cuts $\alpha$ into two subpaths, $\alpha^{-}$and $\alpha^{+}$, whose lengths differ by at most 1 . By (1), $\mathscr{L}(x, y) \subseteq N(\mathscr{L}(x, z) \cup \mathscr{L}(z, y), h)$. We now choose near midpoints of each of the paths $\alpha^{+}$and $\alpha^{-}$and then continue inductively. After at most $p-1$ steps, we see that $\mathscr{L}(x, y) \subseteq N\left(\bigcup_{i=0}^{l-1} \mathscr{L}\left(x_{i}, x_{i+1}\right),(p-1) h\right)$ where $x=x_{0}, x_{1}, \ldots, x_{l}=y$ is the sequence of vertices along $\alpha$. Applying (2) now gives $\mathscr{L}(x, y) \subseteq N(\alpha, p h)$, and so $f(n) \leq p h$ as claimed.

In fact, we aim to show that $f(n)$ is bounded purely in terms of $h$. We proceed as follows.

Let $t=f(n)+2 h+1$. Choose any $w \in \mathscr{L}(x, y)$. Let $l_{0}=\max \{0, d(w, x)-t\}$ and $l_{1}=\max \{0, d(w, y)-t\}$. Since $l=d(x, y)$, we have $l \leq l_{0}+l_{1}+2 t$, and so we can find vertices $x^{\prime}, y^{\prime}$ in $\alpha$ cutting it into subpaths $\alpha=\alpha_{0} \cup \delta \cup \alpha_{1}$, where $d\left(x, x^{\prime}\right) \leq l_{0}, d\left(x^{\prime}, y^{\prime}\right) \leq 2 t$, and $d\left(y^{\prime}, y\right) \leq l_{1}$. If $x=x^{\prime}$ we leave out $\alpha_{0}$, and/or if $y=y^{\prime}$ we leave out $\alpha_{1}$. (We can always assume that $x^{\prime} \neq y^{\prime}$.)

Note that $d\left(w, \alpha_{0}\right) \geq d(w, x)-d\left(x, x^{\prime}\right) \geq d(w, x)-l_{0}$. Therefore, if $x \neq x^{\prime}$, then $l_{0}=d(w, x)-t$, and so $d\left(w, \alpha_{0}\right) \geq t$. But $d\left(x, x^{\prime}\right) \leq d(x, y) \leq n$ and so $\mathscr{L}\left(x, x^{\prime}\right) \subseteq N\left(\alpha_{0}, f(n)\right)$. It follows that $d\left(w, \mathscr{L}\left(x, x^{\prime}\right)\right) \geq t-f(n)=2 h+1$. In other words, if $x \neq x^{\prime}$, then $d\left(w, \mathscr{L}\left(x, x^{\prime}\right)\right) \geq 2 h+1$. Similarly, if $y \neq y^{\prime}$, then $d\left(w, \mathscr{L}\left(y^{\prime}, y\right)\right) \geq 2 h+1$. But

$$
w \in \mathscr{L}(x, y) \subseteq N\left(\mathscr{L}\left(x, x^{\prime}\right) \cup \mathscr{L}\left(x^{\prime}, y^{\prime}\right) \cup \mathscr{L}\left(y^{\prime}, y\right), 2 h\right)
$$


and so $d\left(w, \mathscr{L}\left(x^{\prime}, y^{\prime}\right)\right) \leq 2 h$. Now $d\left(x^{\prime}, y^{\prime}\right) \leq 2 t$ and so $\mathscr{L}\left(x^{\prime}, y^{\prime}\right) \subseteq N(\delta, f(2 t))$. Thus, $w \in N(\delta, f(2 t)+2 h) \subseteq N(\alpha, f(2 t)+2 h)$. Since $w$ was an arbitrary point of $\mathscr{L}(x, y)$, it follows that

$$
f(n) \leq f(2 t)+2 h=f(2 f(n)+4 h+2)+2 h .
$$

Writing $F(n)=2 f(n)+4 h+2$, we have shown that $F(n) \leq F(F(n))+4 h$ for all $n$.

Now, from the earlier claim,

$$
F(n) \leq 2\left(\left(2+\log _{2} n\right) h\right)+4 h+2=2 h\left(4+\log _{2} n\right)+2 .
$$

Suppose $m$ is as in the statement of the theorem. Writing $r=m+2$, we have $2 h(6+\log r)+2 \leq r$, and so $F(n)+4 h \leq 2 h\left(6+\log _{2} n\right)+2<n$ for any $n>r$.

In summary, we have shown that

$$
F(n) \leq F(F(n))+4 h
$$

for all $n$, and that

$$
F(n)+4 h<n
$$

for all $n>r$. It follows that $F(n) \leq r$ for all $n$ (otherwise, we have the contradiction $F(n) \leq F(F(n))+4 h<F(n))$. It now follows that $f(n) \leq s$, where $s=(r / 2)-2 h-1=(m / 2)-2 h$.

We have shown that for all $x, y \in V(G)$ and $\alpha \in \Phi(x, y)$, we have $\mathscr{L}(x, y) \subseteq$ $N(\alpha, s)$. It now follows also that $\alpha \subseteq N(\mathscr{L}(x, y), 2 s)$. Since if $w \in \alpha$, then $w$ cuts $\alpha$ into two subpaths, $\alpha^{-}$and $\alpha^{+}$. Since $\mathscr{L}(x, y)$ is connected and contains $x, y$, we can find some $v \in \mathscr{L}(x, y)$ and $v^{ \pm} \in \alpha^{ \pm}$with $d\left(v, v^{ \pm}\right) \leq s$. Now $d\left(w,\left\{v^{-}, v^{+}\right\}\right) \leq s$, so $d(v, w) \leq 2 s$. We deduce that $d(w, \mathscr{L}(x, y)) \leq 2 s$ as required.

Now suppose that $x, y, z \in V(G)$ and that $\alpha \in \mathscr{I}(x, y), \beta \in \mathscr{I}(x, z)$, and $\gamma \in \mathscr{I}(y, z)$. We have

$$
\alpha \subseteq N(\mathscr{L}(x, y), 2 s) \subseteq N(\mathscr{L}(x, z) \cup \mathscr{L}(z, y), 2 s+h) \subseteq N(\beta \cup \gamma, k),
$$

where

$$
k=3 s+h \leq 3((r / 2)-2 h-1)+h=(3 m-10 h) / 2 .
$$

Thus, $G$ is $k$-hyperbolic.

\section{Estimation of constants}

Given Proposition 3.1 of this paper, we can extract information more efficiently from [B], and bypass much of the proof of Theorem 1.1. Given $\alpha, \beta \in V(\mathscr{G}(g, p))$ with $d(\alpha, \beta) \geq 2$ and $t \in \mathbb{R}$, let $\Lambda_{\alpha \beta}(t)=L\left(\left(e^{t} / \iota\right) \alpha,\left(e^{-t} / \iota\right) \beta, R\right)$, where $\iota=\iota(\alpha, \beta)>0$.

Now, $\iota\left(\left(e^{t} / \iota\right) \alpha,\left(e^{-t} / \iota\right) \beta\right)=1$. Therefore if $2 g+p \geq 195$, then by Lemma 2.4, $\Lambda_{\alpha \beta}(t) \neq \varnothing$. Let $\mathscr{L}(\alpha, \beta)(t)$ be the full subgraph of $\mathscr{G}$ with vertex set $\Lambda_{\alpha \beta}(t)$. It is not 
hard to see that $\mathscr{L}(\alpha, \beta)(t)$ is connected. (For example, the standard argument, going back to work of Lickorish, for showing that $\varphi$ itself is connected effectively does this. This involves interpolating between two curves by a series of surgery operations, see Lemma 1.3 of [B] for example. These can only decrease the intersection number with any fixed curve.) It follows easily that $\mathscr{L}(\alpha, \beta)=\bigcup_{t \in \mathbb{R}} \mathscr{L}(\alpha, \beta)(t)$ is connected. Note that the vertex set of $\mathscr{L}(\alpha, \beta)$ is the "line" $\Lambda_{\alpha \beta}=\bigcup_{t \in \mathbb{R}} \Lambda_{\alpha \beta}(t)$ as defined in [B]. Note also that $\alpha, \beta \in \Lambda_{\alpha \beta}$. If $d(\alpha, \beta) \leq 1$, we set $\Lambda_{\alpha \beta}=\{\alpha, \beta\}$, so that $\mathscr{L}(\alpha, \beta)$ is a single vertex or edge.

We can now verify that the collection $(\mathscr{L}(\alpha, \beta))_{\alpha, \beta \in V(\mathscr{G})}$ satisfies the hypotheses of Proposition 3.1 here with $h=40$. Condition (2) is immediate. For condition (1), let $\alpha, \beta, \gamma \in V(G)$. If these three curves all pairwise intersect, then we set $\tau=$ $\frac{1}{2} \log _{e}(\iota(\alpha, \beta) \iota(\alpha, \gamma) / \iota(\beta, \gamma))$. As in Lemma 4.5 of [B], we see that if $t \leq \tau$, the diameter of $\mathscr{L}(\alpha, \beta)(t) \cup \mathscr{L}(\alpha, \gamma)(t)$ is at most 40 (since we can set $D=20$ ). Similarly, if $t \geq \tau$ then $\mathscr{L}(\alpha, \beta)(t) \cup \mathscr{L}(\beta, \gamma)(t)$ has diameter at most 40. Thus, $\mathscr{L}(\alpha, \beta) \subseteq N(\mathscr{L}(\alpha, \gamma) \cup \mathscr{L}(\gamma, \beta), h)$ with $h=40$. The cases where at least two of the curves $\alpha, \beta, \gamma$ are disjoint follow from a slight modification of this argument, as in [B]. This now gives $m \leq 1320$ and $k \leq 1780$. This shows that if $2 g+p \geq 195$, then $\mathscr{G}(p, q)$ is 1780 -hyperbolic.

In fact, since we are now only using Lemma 4.3 of [B], we can replace $2 R$ by $R$ in Lemma 2.5 here, so that the requirement $16\left(2 R^{2}\right) \leq \xi_{0}^{5}$ becomes $16 R^{2} \leq \xi_{0}^{5}$, and so we can replace the resulting factor of 512 in $(*)$ by 256 . It is therefore sufficient that $2 g+p \geq 107$. We have shown that if $2 g+p \geq 107$, then $\mathscr{G}(g, p)$ is 1780-hyperbolic.

We can deal with lower complexity surfaces using larger values of $q$ from Corollary 2.2. In general, we require that

$$
2^{q+4}(2 g+p-1)^{2}(2 g+p+6)^{2} \leq \pi(2 g+p-4)^{q+1} .
$$

For example, with $q=5$, this is satisfied for $2 g+p \geq 26$. This gives

$$
D=4(q+1)=24, \quad h=2 D=48, \quad m \leq 1584, \quad k \leq 2136 .
$$

In other words, if $2 g+p \geq 26$, then $\mathscr{G}(g, p)$ is 2064-hyperbolic. Similarly (with $q=6)$, if $2 g+p \geq 14$, then $\mathscr{G}(g, p)$ is 2492-hyperbolic, and so on.

For the cases where $2 g+p \leq 6$, we need to revert to previous arguments. The estimates and methods in [Tang 2013] might give improvements for some of the lower complexities.

There is scope for other improvements in various directions. For the bounds on complexity for example, suppose $p=0$. In the proof of Lemma 2.3 we don't have to worry about trivial regions, so we can easily obtain $l \leq 2 \lambda$, allowing us to reset $\xi_{2}=2 g+2$. We can also reset $\xi_{1}=2 g$. For Corollary 2.2, we could set $h=1 / 4 \pi$, further decreasing $R$ by a factor of $\sqrt{2}$. In Lemma 1.3 of [B], 
we can eliminate the factor of 2 in the hypotheses, and thereby weaken those of Corollary 2.2 here to saying that $\iota(\alpha, \beta) \leq x_{0}^{q}$. The fact that we have replaced $2 R$ by $R$ also gives us another factor of 2 , so that our requirement, when $q=4$, now becomes $R^{2} \leq \xi_{0}^{5}$. Together these now give $8(2 g)^{2}(2 g+2)^{2} \leq \pi(2 g-4)^{5}$, that is, $4 g^{2}(g+1)^{2} \leq \pi(g-2)^{5}$, which holds for $g \geq 8$. In other words, $\varphi(g, 0)$ is 1780-hyperbolic for $g \geq 8$.

We remark that in [Hensel, Przytycki and Webb 2013], it is shown that every curve graph is "17-hyperbolic" in the sense that, for every geodesic triangle, there is a vertex at a distance of no more than 17 from each of its sides. From this, one can easily derive a uniform hyperbolicity constant in the sense we have defined it.

\section{References}

[Aougab 2013] T. Aougab, "Uniform hyperbolicity of the graphs of curves", Geom. Topol. 17:5 (2013), 2855-2875. MR 3190300 Zbl 1273.05050

[Bowditch 2006] B. H. Bowditch, "Intersection numbers and the hyperbolicity of the curve complex", J. Reine Angew. Math. 598 (2006), 105-129. MR 2009b:57034 Zbl 1119.32006

[Clay, Rafi and Schleimer 2013] M. Clay, K. Rafi, and S. Schleimer, "Uniform hyperbolicity of the curve graph via surgery sequences", preprint, 2013. arXiv 1302.5519

[Gilman 2002] R. H. Gilman, "On the definition of word hyperbolic groups", Math. Z. 242:3 (2002), 529-541. MR 2004b:20062 Zbl 1047.20033

[Gromov 1987] M. Gromov, "Hyperbolic groups", pp. 75-263 in Essays in group theory (Berkeley, CA, 1985), edited by S. M. Gersten, Math. Sci. Res. Inst. Publ. 8, Springer, New York, 1987. MR 89e:20070 Zbl 0634.20015

[Harvey 1981] W. J. Harvey, "Boundary structure of the modular group", pp. 245-251 in Riemann surfaces and related topics (Stony Brook, NY, 1978), edited by I. Kra and B. Maskit, Ann. of Math. Stud. 97, Princeton University Press, 1981. MR 83d:32022 Zbl 0461.30036

[Hensel, Przytycki and Webb 2013] S. Hensel, P. Przytycki, and R. C. H. Webb, "1-slim triangles and uniform hyperbolicity for arc graphs and curve graphs", preprint, 2013. To appear in J. Eur. Math. Soc. arXiv 1301.5577

[Masur and Minsky 1999] H. A. Masur and Y. N. Minsky, "Geometry of the complex of curves, I: Hyperbolicity”, Invent. Math. 138:1 (1999), 103-149. MR 2000i:57027 Zbl 0941.32012

[Masur and Minsky 2000] H. A. Masur and Y. N. Minsky, "Geometry of the complex of curves, II: Hierarchical structure”, Geom. Funct. Anal. 10:4 (2000), 902-974. MR 2001k:57020 Zbl 0972.32011

[Masur and Schleimer 2013] H. A. Masur and S. Schleimer, "The geometry of the disk complex", J. Amer. Math. Soc. 26:1 (2013), 1-62. MR 2983005 Zbl 1272.57015

[Tang 2013] R. Tang, Covering maps and hulls in the curve complex, thesis, University of Warwick, Coventry, 2013.

[Webb 2013] R. C. H. Webb, "Uniform bounds for bounded geodesic image theorems", preprint, 2013. To appear in J. Reine Angew. Math. arXiv 1301.6187

[Weil 1926] A. Weil, "Sur les surfaces à courbure négative", C. R. Acad. Sci. (Paris) 182 (1926), 1069-1071. JFM 52.0712.05 
Received March 8, 2013. Revised June 24, 2013.

BRIAN H. BOWDITCH

MATHEMATiCs Institute

UNIVERSITY OF WARWICK

COVENTRY, CV4 7AL

UNITED KINGDOM 


\title{
PACIFIC JOURNAL OF MATHEMATICS
}

\author{
msp.org/pjm
}

Founded in 1951 by E. F. Beckenbach (1906-1982) and F. Wolf (1904-1989)

\section{EDITORS}

Don Blasius (Managing Editor)

Department of Mathematics

University of California

Los Angeles, CA 90095-1555

blasius@math.ucla.edu

\author{
Paul Balmer \\ Department of Mathematics \\ University of California \\ Los Angeles, CA 90095-1555 \\ balmer@math.ucla.edu \\ Robert Finn \\ Department of Mathematics \\ Stanford University \\ Stanford, CA 94305-2125 \\ finn@math.stanford.edu \\ Sorin Popa \\ Department of Mathematics \\ University of California \\ Los Angeles, CA 90095-1555 \\ popa@math.ucla.edu
}

\author{
Vyjayanthi Chari \\ Department of Mathematics \\ University of California \\ Riverside, CA 92521-0135 \\ chari@math.ucr.edu \\ Kefeng Liu \\ Department of Mathematics \\ University of California \\ Los Angeles, CA 90095-1555 \\ liu@math.ucla.edu \\ Jie Qing \\ Department of Mathematics \\ University of California \\ Santa Cruz, CA 95064 \\ qing@ cats.ucsc.edu
}

\section{PRODUCTION}

Silvio Levy, Scientific Editor, production@msp.org

\section{SUPPORTING INSTITUTIONS}

ACADEMIA SINICA, TAIPEI

CALIFORNIA INST. OF TECHNOLOGY

INST. DE MATEMÁTICA PURA E APLICADA

KEIO UNIVERSITY

MATH. SCIENCES RESEARCH INSTITUTE

NEW MEXICO STATE UNIV.

OREGON STATE UNIV.

\author{
STANFORD UNIVERSITY \\ UNIV. OF BRITISH COLUMBIA \\ UNIV. OF CALIFORNIA, BERKELEY \\ UNIV. OF CALIFORNIA, DAVIS \\ UNIV. OF CALIFORNIA, LOS ANGELES \\ UNIV. OF CALIFORNIA, RIVERSIDE \\ UNIV. OF CALIFORNIA, SAN DIEGO \\ UNIV. OF CALIF., SANTA BARBARA
}

\author{
Daryl Cooper \\ Department of Mathematics \\ University of California \\ Santa Barbara, CA 93106-3080 \\ cooper@math.ucsb.edu \\ Jiang-Hua Lu \\ Department of Mathematics \\ The University of Hong Kong \\ Pokfulam Rd., Hong Kong \\ jhlu@maths.hku.hk \\ Paul Yang \\ Department of Mathematics \\ Princeton University \\ Princeton NJ 08544-1000 \\ yang@math.princeton.edu
}

These supporting institutions contribute to the cost of publication of this Journal, but they are not owners or publishers and have no responsibility for its contents or policies.

See inside back cover or msp.org/pjm for submission instructions.

The subscription price for 2014 is US $\$ 410 /$ year for the electronic version, and \$535/year for print and electronic.

Subscriptions, requests for back issues and changes of subscribers address should be sent to Pacific Journal of Mathematics, P.O. Box 4163, Berkeley, CA 94704-0163, U.S.A. The Pacific Journal of Mathematics is indexed by Mathematical Reviews, Zentralblatt MATH, PASCAL CNRS Index, Referativnyi Zhurnal, Current Mathematical Publications and Web of Knowledge (Science Citation Index).

The Pacific Journal of Mathematics (ISSN 0030-8730) at the University of California, c/o Department of Mathematics, 798 Evans Hall \#3840, Berkeley, CA 94720-3840, is published twelve times a year. Periodical rate postage paid at Berkeley, CA 94704, and additional mailing offices. POSTMASTER: send address changes to Pacific Journal of Mathematics, P.O. Box 4163, Berkeley, CA 94704-0163.

PJM peer review and production are managed by EditFLOW ${ }^{\circledR}$ from Mathematical Sciences Publishers.

\section{PUBLISHED BY}

\section{mathematical sciences publishers \\ nonprofit scientific publishing}

http://msp.org/

(C) 2014 Mathematical Sciences Publishers 


\section{PACIFIC JOURNAL OF MATHEMATICS}

Volume $269 \quad$ No. $2 \quad$ June 2014

Totaro's question for simply connected groups of low rank

257

JODI BLACK and RAMAN PARIMALA

Uniform hyperbolicity of the curve graphs

269

BRIAN H. BOWDITCH

Constant Gaussian curvature surfaces in the 3-sphere via loop groups

281

DAVID BRANDER, JUN-ICHI INOGUCHI and SHIMPEI KOBAYASHI

On embeddings into compactly generated groups

305

PIERRE-EMmanuel CAPRACE and Yves CORNULIER

Variational representations for $N$-cyclically monotone vector fields

ALFRED GALICHON and NASSIF GHOUSSOUB

Restricted successive minima

341

MaRTIN HenK and CARSTEN THIEL

Radial solutions of non-Archimedean pseudodifferential equations

355

ANATOLY N. KochubeI

A Jantzen sum formula for restricted Verma modules over affine Kac-Moody

algebras at the critical level

JOHANNES KÜBEL

Notes on the extension of the mean curvature flow

YAN LENG, ENTAO ZHAO and HaORAN ZHAO

Hypersurfaces with prescribed angle function

Henrique F. DE Lima, ERAldo A. Lima JR. and Ulisses L. Parente

Existence of nonparametric solutions for a capillary problem in warped products

JoRGE H. LiRA and GABRIELA A. WANDERLEY

A counterexample to the simple loop conjecture for $\operatorname{PSL}(2, \mathbb{R})$

425

KATHRYN MANN

Twisted Alexander polynomials of 2-bridge knots for parabolic representations

TAKAYUKI MORIFUJI and ANH T. TRAN

Schwarzian differential equations associated to Shimura curves of genus zero

FANG-TING TU

Polynomial invariants of Weyl groups for Kac-Moody groups 